\title{
EVALUACIÓN DE LA CALIDAD DEL SERVICIO PERCIBIDA EN ENTIDADES BANCARIAS A TRAVÉS DE LA ESCALA SERVQUAL
}

\section{EVALUATION OF PERCEIVED SERVICE QUALITY IN BANKS USING THE SERVQUAL SCALE}

\author{
Roxana González Álvarez \\ Fecha de recepción: 19 de Noviembre de 2014 \\ Fecha de aprobación: 16 de Marzo de 2015
}

Referencia: R. González Álvarez. (2015). Evaluación de la calidad del servicio persivida en entidades Bancarias a través de la escala Servqual. Ciencia e Ingeniería Neogranadina, 25 (1), pp. 113 - 135

\section{RESUMEN}

El presente trabajo se realizó en una sucursal bancaria en la provincia de Cienfuegos, Cuba, durante los últimos tres trimestres del año 2013 con el objetivo fundamental de evaluar y mejorar la calidad percibida con un enfoque de gestión por procesos y mejora continua. Para ello, se diseñó e implementó un procedimiento para la evaluación de la calidad percibida de los servicios bancarios basado en la escala multidimensional Servqual. Para la recopilación de información se utilizaron técnicas tales como: entrevistas, tormenta de ideas, revisión de documentos, trabajo con expertos, trabajo en equipo y observación directa. Se hizo uso de herramientas clásicas de la calidad y de gestión de procesos, así como la técnica $5 \mathrm{~W}$ y $1 \mathrm{H}$, y la metodología de Proceso Esbelto. Como resultados fundamentales de la investigación se identificaron las principales brechas de insatisfacción en la calidad del servicio a partir de las percepciones de los clientes, así como la obtención de una calificación global de la calidad del servicio. Se determinó que para las 22 declaraciones del cuestionario Servqual las percepciones de los clientes no superan las expectativas. Las brechas con menor índice de calidad son aquellas que se relacionan con el tiempo de servicio, por lo que se propone un conjunto de acciones que contribuyan a la mejora de la calidad percibida.

Palabras claves: Calidad de servicio, gestión por procesos, mejora continua, metodología Servqual, servicios bancarios.

1. Ingeniera Industrial, M.Sc. en Ingeniería Industrial, Mención Calidad, Profesor Asistente. Universidad de Cienfuegos, Cienfuegos, Cuba, rgonzalez@ucf.edu.cu 


\section{ABSTRACT}

The research presented in this paper was developed in a banking branch in the province of Cienfuegos, Cuba, during the last three quarters of 2013, with the main objective of evaluating and improving the perceived quality while focusing on process management and continuous improvement. For this purpose, a procedure to evaluate the perceived quality of banking services based on in the multi-dimensional Servqual scale was designed and implemented. Data collection was accomplished by the use of different techniques, such as interviews, brainstorming, review of documents, working with experts, teamwork and direct observation. Classical tools of quality and process management were used, as well as the $5 \mathrm{Ws}$ and $1 \mathrm{H}$ technique, and the methodology of Lean Process. The results of the research led to the identification of the main gaps of dissatisfaction with the service quality from the perceptions of customers, as well as to the completion of an assessment of overall service. It was determined that for the 22 statements of the Servqual questionnaire, customer perceptions did not exceed expectations. The gaps with lower quality index are those related to the length of service, for which a set of actions that contribute to improving the perceived quality are proposed.

Keywords: Banking services, continual improvement, process management, service quality, Servqual methodology.

\section{INTRODUCCIÓN}

Un servicio bancario es la actividad que desarrollan las entidades financieras como empresas de servicios; es decir, todas aquellas acciones que se establecen para servir a los clientes [1]. Los servicios bancarios son complejos al combinar aspectos tangibles (pagos, retiros, depósitos y papelería) y aspectos intangibles (sensación de seguridad, capacidad de respuesta y responsabilidad de los funcionarios) [2], y se caracterizan por sufrir rápidos cambios en su ambiente [3], algunos gracias a la tecnología, y que, relacionados con la información, resultan ser cada vez más importantes [4].

La globalización ha llevado a dichas instituciones a ser competitivas con inversión tecnológica e innovación como estrategias de diferenciación para asegurar la fidelidad de sus clientes [5], pero estas son de fácil transferencia, e incluso superadas por la competencia [6]. Es por ello que estas instituciones deben enfatizar en lo intangible del servicio, que agrega valor para desarrollar y mantener sus ventajas competitivas, si se toma en cuenta que "los servicios son lo único que los bancos pueden vender, son la única manera de ser diferentes" [7]. Además, los productos bancarios son básicamente los mismos por lo que el único elemento que permite lograr diferenciación es la calidad del servicio con la que ofrecen sus productos a su cartera de clientes.

La calidad del servicio se define de manera general como una evaluación de la calidad desde la percepción de los clientes, donde estos comparan el servicio que esperan con las percepciones del servicio que ellos reciben. Toda calidad es percibida por alguien [8-9]. 
Este hecho señala la importancia de centrarse en el cliente, como único juez y evaluador de la calidad del servicio, cuyos criterios deben considerarse para garantizar la mejora continua de los procesos. La orientación al cliente es un elemento crítico, puesto que este es activador en materia de servicio. Dada su demanda es que dichos procesos se realizan, está presente en la producción, los recibe y evalúa según criterios personales en el tiempo en que se efectúan.

La mayoría de las investigaciones en el sector bancario en relación a la temática que aquí se aborda se han fundamentado en el uso o adaptación de escalas ampliamente validadas como el Servqual [10-11] y el Servperf [12-13] o en definir las dimensiones de la calidad del servicio y construir un modelo para medirla y luego mejorarla [1415]. También se han realizado estudios teniendo en cuenta el modelo de la imagen de Grönroos en combinación con la escala Servqual [16].

De todos estos modelos, el Servqual ha sido el método de investigación más nombrado y utilizado tanto en el mundo académico como empresarial [17]. Esta metodología entiende la calidad del servicio como una función de la discrepancia entre las expectativas de los consumidores sobre el servicio que van a recibir y sus percepciones sobre el servicio prestado por la organización [14].

Deesta manera, el Servqual permite determinar lo que desean los clientes de la organización y lo que perciben encontrar, así como las brechas de insatisfacción específicas y el orden de los vacíos de calidad, desde el más grave y urgente hasta el menos grave [14].
Pese a que se identifican en la literatura consultada una gran variedad de estudios relacionados con la calidad del servicio en las instituciones bancarias, se carece de procedimientos que orienten la acción de evaluación de forma sistemática para la mejora de la calidad percibida con un enfoque a procesos y de mejora continua. Socializar las prácticas que están siendo desarrolladas y de resultados positivos en relación con esta temática es la principal intención de la investigación que se presenta, considerando el diseño y aplicación de un procedimiento para la evaluación de la calidad percibida de los servicios bancarios.

\section{MATERIALES Y MÉTODOS}

El procedimiento para la evaluación de la calidad percibida de los servicios bancarios se fundamenta en la escala multidimensional Servqual propuesta por Parasuraman, Berry y Zeithaml [18] y toma como base los procedimientos para la medición de la calidad percibida de Díaz y Pons [19], Moreno [20] y Curbelo [21]. La propuesta introduce la gestión por procesos y el ciclo de mejora continua Planificar-Hacer-Verificar-Actuar basándose en el procedimiento de gestión de procesos propuesto por Beltrán, Carmona, Carrasco, Rivas y Tejedor [22], elementos que no se identifican en estudios anteriores y que contribuyen a la mejora de la calidad y del valor percibido por el cliente.

La propuesta está dirigida a:

- Describir y documentar el proceso de servicio bancario objeto de estudio.

- Obtener una calificación global de la calidad del servicio.

- Determinar las principales brechas de 
insatisfacción en la calidad del servicio según las percepciones de los clientes.

- Proponer un conjunto de acciones encaminadas a la mejora del proceso de servicio en las que se evalúa la calidad percibida a partir de la aplicación de un conjunto de técnicas y herramientas para el mejoramiento continuo.

- Comparar el desempeño de las diversas sucursales bancarias desde la percepción de los clientes.

Un elemento a destacar es que el procedimiento toma en cuenta la participación de los trabajadores en la búsqueda y propuesta de acciones de mejora. Este hecho permite que el personal entienda su papel en el logro de las metas y que se sienta parte de este proceso para ir creando una cultura de calidad. Otro elemento a destacar es que considera la capacitación del equipo de trabajo para garantizar el adecuado uso de herramientas y técnicas que contribuyen a la evaluación de la calidad percibida de los servicios bancarios. Además da la posibilidad de que cada cliente del banco opine del servicio que recibe teniendo en cuenta diferentes criterios, de modo que considere que sus opiniones son en realidad escuchadas para mejorar la calidad de los servicios, lo que puede percibir a su regreso a la institución bancaria o por referencia de otros clientes.

El procedimiento concibe los siguientes principios:

- Enfoque a procesos: Las instituciones bancarias deben seguir el enfoque por procesos. La descripción del proceso de servicios que evaluará el cliente es el punto de partida para entender y visualizar de manera global en qué consiste el mismo dejando clara su trayectoria desde el inicio hasta el fin.
- Enfoque a servicios: El procedimiento es aplicable a organizaciones netamente de servicios, en particular se orienta a las instituciones bancarias.

- Enfoque a clientes: Los clientes de los servicios de las instituciones bancarias serán responsables de evaluar la calidad del servicio y, en función de su percepción, se identificarán oportunidades de mejora para garantizar un incremento de la calidad de servicio percibida.

- Enfoque basado en hechos para la toma de decisión: Las propuestas de acciones para la mejora de los procesos de servicios bancarios estarán basadas en los resultados de la evaluación de la calidad percibida.

- Mejora continua: Al enfocar las mejoras se tiene en cuenta la retroalimentación permanente a la organización para garantizar que los clientes de las instituciones bancarias se mantengan satisfechos. El objetivo primero y fundamental es mejorar para dar al cliente el mayor valor agregado, mediante una mejora continua y sistemática de la calidad percibida.

- Participación del personal: El personal, a todos los niveles, es la esencia de una organización, y su total compromiso posibilita que sus habilidades sean usadas para el beneficio de la organización. Un sistema exitoso de mejoramiento continuo requiere descansar en la habilidad de los miembros de una organización para reconocer oportunidades de mejoramiento. En resumidas cuentas, las personas son la fuente del mejoramiento.

- Enfoque sistémico: Cualquiera de los servicios en que se aplique el procedimiento se integra al sistema bancario, como tal su relación con otras partes componentes del mismo queda declarada al efectuar la evaluación de la calidad percibida. 
- Aplicabilidad: El procedimiento queda lo suficientemente detallado y con las herramientas necesarias para su aplicación en instituciones bancarias, lo que permitirá comparar el desempeño de las diversas sucursales e incluso con la competencia.

- Adaptabilidad: El procedimiento es adaptable a cualquier servicio de los que se brindan en una institución bancaria.

- Pertinencia: El procedimiento está diseñado para una aplicación constante en instituciones bancarias que garantice conocer el estado de opinión de los clientes sobre los servicios que recibe.

- Flexibilidad: El procedimiento es flexible en cuanto a las herramientas que se proponen para la consecución de cada una de las etapas y pasos.

- Consistencia lógica: El procedimiento se articula de una forma lógica que permite cumplir con los principios anteriores, por lo que está compuesto de etapas y pasos, en los que se cohesionan criterios relacionados con calidad percibida, su evaluación y mejora.

- Aprendizaje: En su concepción, el procedimiento contempla la creación de un equipo de trabajo y su capacitación en temáticas relacionadas con la calidad del servicio y su medición, la gestión por procesos y las técnicas a aplicar.

Cada uno de estos principios hace que la propuesta que se especifica en esta investigación tenga características particulares en comparación con otras analizadas.

El procedimiento se muestra en la Figura 1. Este se estructuró metodológicamente en cuatro etapas y trece pasos. Para cada una de las etapas se declaran los objetivos a cumplir y las posibles herramientas a utilizar.

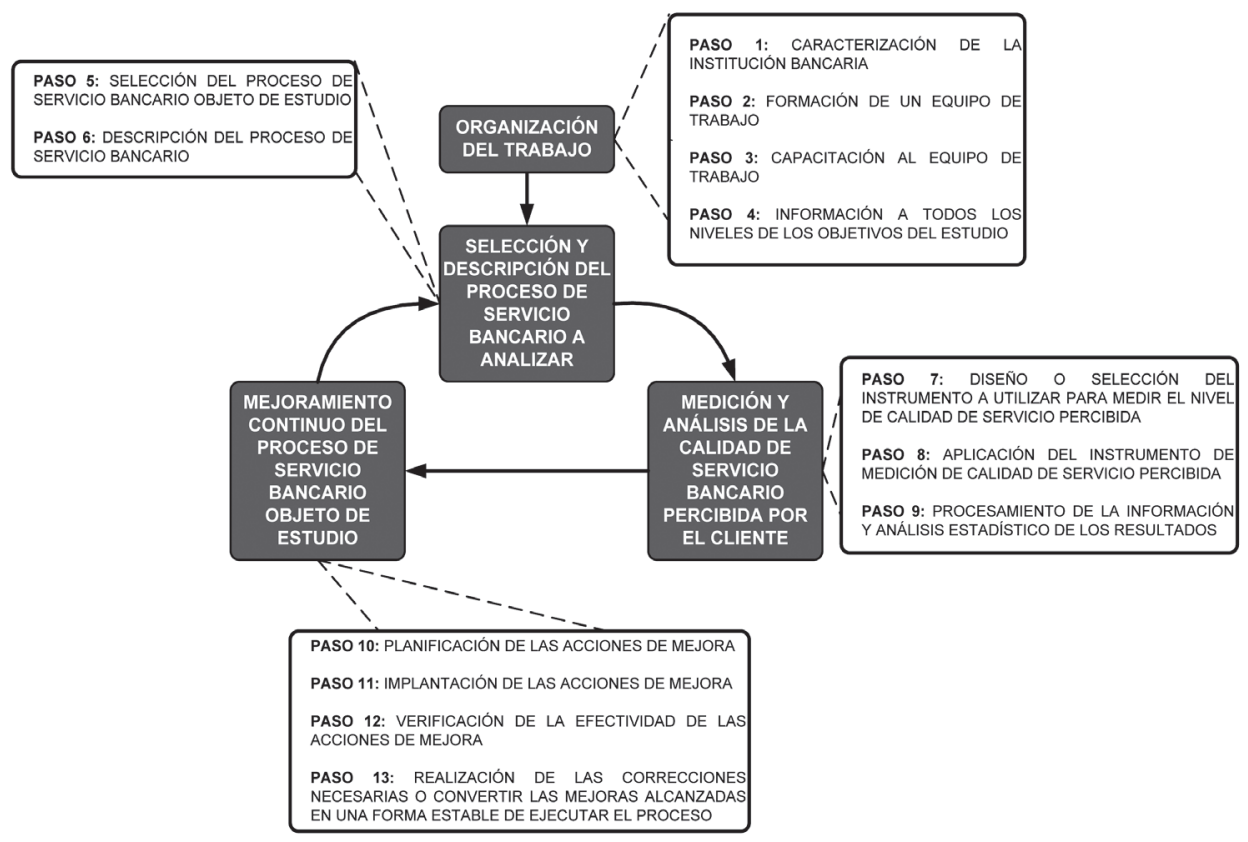

Figura 1. Procedimiento para la evaluación de la calidad percibida de los servicios bancarios. 


\section{Etapa I: Organización del trabajo}

Objetivos:

1. Caracterizar la institución bancaria objeto de estudio.

2. Conformar un equipo de trabajo.

3. Dar capacitación al equipo de trabajo encargado de tomar las decisiones relativas al estudio de calidad de servicio percibida que se realiza.

Herramientas:

- Entrevistas.

- Revisión de documentos.

- Reuniones participativas.

- Mapa general de procesos.

Paso 1. Caracterización de la institución bancaria

En este paso se recopila toda la información necesaria referente a la institución bancaria objeto de estudio de manera que se puedan conocer sus principales características.

Paso 2. Formación de un equipo de trabajo

El equipo de trabajo debe estar integrado por un grupo de expertos conocedores del tema e interesados en el mismo, de forma tal que aporten información precisa, participen en todas las etapas de la investigación, y puedan tomar las decisiones convenientes. Se deberá para ello calcular el número de expertos necesarios y para su selección utilizar los siguientes criterios:

- Conocimiento del tema a tratar.

- Capacidad para trabajar en equipo y espíritu de colaboración.

- Años de experiencia en el cargo.

- Vinculación directa a la actividad.
Se debe calcular además el coeficiente de competencia para cada uno de ellos según la metodología de Cortés e Iglesias [23] con el objetivo de asegurar que los expertos que se consulten puedan aportar criterios significativos respecto al tema objeto de estudio. Se seleccionan aquellos expertos que tengan un coeficiente de competencia entre medio y alto.

\section{Paso 3. Capacitación al equipo de trabajo}

Se debe realizar una capacitación al equipo de trabajo para que sus miembros conozcan y entiendan de manera colectiva los principales elementos relacionados con la calidad de los servicios y los modelos para su medición, la gestión por procesos, así como las herramientas a utilizar. Este paso facilita la correcta ejecución de la evaluación.

Paso 4. Información a todos los niveles de los objetivos del estudio

Dado que las instituciones bancarias son organizaciones netamente de servicios y tomando en cuenta las características diferenciales de este sector, se debe lograr una participación activa de los trabajadores, puesto que ellos son los actores sociales de los procesos que se desarrollan en la organización. Muchos de ellos tienen contacto directo con los clientes (personal en contacto), de manera que pueden aportar criterios de mejora efectivas que contribuyan a elevar el nivel de calidad del servicio percibido por el cliente. Se debe realizar una reunión con los trabajadores de las áreas implicadas o con todos los de la organización en dependencia del alcance del estudio y de las características de esta, donde se explique la necesidad de su participación activa en el mismo, buscando compromiso y 
contribución con su desarrollo exitoso. Se les dan a conocer los objetivos que se persiguen, que pueden rediseñarse $o$ ampliarse a partir de la retroalimentación.

\section{Etapa II: Selección y descripción del proceso de servicio bancario a analizar}

Objetivo: Documentar el proceso de servicio bancario objeto de estudio.

Herramientas:

- Dinámicas de equipos de trabajo.

- Revisión y análisis de documentos.

- Entrevistas.

- Método de expertos.

- Diagrama de Pareto.

- Reuniones participativas.

- Observación directa.

- Diagrama SIPOC.

- Diagrama de flujo.

- Ficha de proceso.

Paso 5. Selección del proceso de servicio bancario objeto de estudio

El equipo de trabajo es el encargado de seleccionar el proceso de servicio bancario objeto de estudio mediante el uso de técnicas como el método de expertos o el diagrama de Pareto a partir de los resultados de auditorías, encuestas, quejas de clientes, entre otros.

Paso 6. Descripción del proceso de servicio bancario

Este paso incluye la descripción de las actividades y de las características del proceso. El producto final esperado de este paso de descripción del proceso es un documento que permite entender y visualizar de manera global en qué consiste el mismo. El mapeo del proceso facilita la visualización de cada una de las operaciones y actividades involucradas, de manera aislada o interrelacionadas. Este flujo detallado deja clara la trayectoria de la actividad desde su inicio hasta su conclusión.

\section{Etapa III: Medición y análisis de la calidad de servicio bancario percibida por el cliente}

Objetivo: Determinar el estado actual de la calidad de servicio bancario percibida por el cliente.

Herramientas:

- Revisión de la literatura.

- Cuestionarios.

- Entrevistas.

- Estadística descriptiva.

- Análisis de fiabilidad.

- Prueba Mann-Whitney para comparar medianas.

Paso 7. Diseño o selección del instrumento a utilizar para medir el nivel de calidad de servicio percibido

El equipo de trabajo es el encargado de diseñar o seleccionar el instrumento más adecuado para medir el nivel de calidad de servicio percibido. Se recomienda la utilización del Cuestionario Servqual. Mediante el trabajo con expertos se adapta y ajusta dicho cuestionario a las características de las instituciones bancarias. El mismo tiene como objetivo general conocer el nivel de calidad percibida que se presenta con respecto a los servicios bancarios. Todas las declaraciones que se encuentran en el cuestionario están enunciadas en sentido positivo en relación con la calidad del servicio. 
Cuenta con tres apartados acompañados de las instrucciones para responderlo: datos generales, evaluación de la calidad percibida de servicios bancarios y sugerencias.

El apartado «Evaluación de la calidad percibida de servicios bancarios» consta a su vez de tres partes, que se deben aplicar en dos momentos diferentes de tiempo. La primera parte capta las expectativas de los clientes y debe ser aplicada antes de recibir el servicio que ofrece el banco; la segunda, evalúa la importancia que tienen cada una de las cinco dimensiones del servicio (elementos tangibles, fiabilidad, capacidad de respuesta, seguridad y empatía). y la tercera evalúa la percepción de los clientes sobre la calidad del servicio brindado por la institución bancaria y debe aplicarse después que el cliente haya recibido el servicio ofrecido por el banco. Tanto para expectativas como para percepciones se utiliza una escala tipo Likert de dimensión siete donde (1) significa estar totalmente en desacuerdo y (7) estar totalmente de acuerdo con una declaración dada.

Paso 8. Aplicación del instrumento de medición de calidad de servicio percibida

En este paso se aplica el instrumento de medición seleccionado o diseñado. Para ello, se debe calcular el tamaño de muestra necesario y determinar el tipo de muestreo a utilizar.

Paso 9. Procesamiento de la información y análisis estadístico de los resultados de la medición de calidad de servicio percibido

El procesamiento de las encuestas se realiza con la ayuda de programas estadísticos como el SPSS Versión 19.0 y el Statgraphics Centurion. Una vez procesada la información, se analizan los resultados obtenidos con vistas a identificar oportunidades de mejora. Según Parasuraman, Berry y Zeithaml [18], la puntuación de Servqual se define como:

Puntuación de Servqual $=$ Percepciones del servicio-Expectativas del servicio (1)

- Puntación de Servqual = 0 Existe calidad en el servicio.

- Puntación de Servqual > 0 Existe un excelente o extraordinario nivel de calidad.

- Puntación de Servqual < 0 Existe déficit o falta de calidad (calidad deficiente).

\section{Etapa IV: Mejoramiento continuo del proceso de servicio bancario objeto de estudio}

Objetivo: Gestionar la mejora del proceso de servicio bancario objeto de estudio mediante la propuesta de acciones de mejora, su implantación y seguimiento.

Herramientas:

- Control estadístico de procesos.

- Diagrama causa-efecto.

- Método Delphi.

- 5W y $1 \mathrm{H}$.

- Metodología de proceso esbelto.

- Diseño de experimentos.

- Tormenta de ideas.

- Benchmarking.

En esta etapa del procedimiento se pretende, a partir de los resultados de la evaluación de la calidad percibida obtenidos en la etapa anterior, proponer acciones para la mejora del proceso de servicio bancario objeto de estudio, implantarlas y dar seguimiento, utilizando para ello el ciclo de mejora continua. Esta etapa se compone a su vez de los siguientes pasos: 
Paso 10. Planificar

El paso de planificación implica establecer qué se quiere alcanzar (objetivos) y cómo se pretende alcanzar (planificación de las acciones). Esta etapa se puede descomponer, a su vez, en las siguientes actividades:

1. Identificación y análisis de la situación.

2. Establecimiento de las mejoras a alcanzar (objetivos).

3. Identificación, selección y programación de las acciones.

Paso 11. Hacer

En este paso se lleva a cabo la implantación de las acciones planificadas según la etapa anterior.

\section{Paso 12. Verificar}

En este paso se verifica la efectividad de las acciones para alcanzar las mejoras planificadas (objetivos).

\section{Paso 13. Actuar}

En función de los resultados de la comprobación anterior, en este paso se realizan las correcciones necesarias (ajuste) o se convierten las mejoras alcanzadas en una forma estable de ejecutar el proceso (actualización).

\section{RESULTADOS Y ANÁLISIS}

A continuación se muestran los resultados del estudio realizado en cada una de las etapas y pasos del procedimiento diseñado, tomando como objeto de estudio una sucursal bancaria perteneciente al Banco Popular de Ahorro (BPA).

\subsection{Etapa I: Organización del trabajo}

Paso 1: Caracterización de la institución bancaria

En la sucursal bancaria se realizan como promedio 1945 operaciones diarias. Tiene a su favor que cuenta con una localización privilegiada, pues se encuentra ubicada en el mismo centro de la ciudad de Cienfuegos, Cuba, donde es de fácil acceso para los clientes de cualquier tipo. La sucursal distingue claramente dos grupos de clientes que forman sus segmentos: la banca particular que la constituyen las personas independientes y la banca estatal que la constituyen las empresas o instituciones que solicitan los servicios de la sucursal. Ha de destacarse que esta institución bancaria mantiene su orientación hacia el segmento de particulares, comenzando apenas a prestar atención al sector corporativo.

Como principal resultado de este paso se obtiene una caracterización general de la organización donde:

- Se elabora el mapa general de procesos con las siguientes clasificaciones: estratégicos, claves y de apoyo. Se identifican dos procesos claves: el de caja y el comercial, siendo estos los que garantizan directamente el cumplimiento de la misión de la organización.

- Se describe el funcionamiento de la sucursal y del flujo de clientes: De este análisis se concluye que el $26 \%$ de los clientes pasan al área comercial y el 74\% lo hacen al área de caja.

- Se identifican los principales productos y servicios que ofrece.

- Se realiza un análisis de las quejas emitidas por los clientes por insatisfacciones en la calidad del servicio correspondientes a 
los últimos tres trimestres del año 2013: se evidencia un incremento significativo en el número de quejas con 6,11 y 21 , respectivamente. No obstante y según los directivos de la sucursal, en la mayoría de las ocasiones el cliente insatisfecho tiende a no expresar su insatisfacción de forma espontánea. Medianteeluso del Diagramade Pareto, quedan estratificadas las quejas por mala calidad en el servicio y se concluye que la mayoría de estas están relacionadas con la demora del servicio y el exceso de tiempo de espera en la fila, que representaron el $31.58 \%$ y $21.05 \%$ del total, respectivamente. Las quejas referidas al espacio limitado $(15.79 \%$ y máquinas bloqueadas $(13.16 \%)$ complementan la representación del 80\% de las quejas de los clientes. Además, en la planeación estratégica de la organización se evidencia, como una de las debilidades identificadas a partir del análisis DOFA (Debilidades-Oportunidades-FortalezasAmenazas), que el servicio brindado no siempre tiene la calidad requerida debido al desconocimiento de las causas que inciden en las insatisfacciones de los clientes, con base en el hecho de que la organización no sabe cómo medirlas.

Lo anterior corrobora la necesidad de evaluar las percepciones que tienen los clientes sobre el servicio brindado por la sucursal, de modo que se puedan identificar las principales brechas de insatisfacción en la calidad del servicio, con el objetivo de proponer acciones encaminadas a la mejora de la calidad de los procesos de servicio bancario.

\section{Paso 2: Formación de un equipo de trabajo}

Con el objetivo de formar el equipo de trabajo se calcula el número de expertos necesarios, que resulta ser 8. Luego se elabora una lista de posibles candidatos a partir de los criterios establecidos en el diseño del procedimiento y del análisis realizado por la directora de la sucursal, quien tiene 15 años de experiencia en el sector:

1. Un profesor de la carrera de Ingeniería Industrial de la Universidad de Cienfuegos.

2. Un estudiante de la carrera de Ingeniería Industrial de la Universidad de Cienfuegos.

3. Directora de la sucursal 4822.

4. Gerente Comercial.

5. Gerente de Efectivo.

6. Gerente de Contabilidad.

7. Gerente de Recuperación.

8. Un trabajador de reconocido prestigio.

El cálculo del coeficiente de competencia se realiza siguiendo el método de Cortés e Iglesias [23] mediante la aplicación de un cuestionario donde se le pide a cada experto autoevaluar en una escala de 0 a 10 sus conocimientos sobre la calidad de los servicios y evaluar la influencia de las seis fuentes de argumentación que establecen los autores en una escala de alto, medio y bajo.

Para el cálculo del coeficiente de competencia se utiliza la fórmula:

$$
\mathrm{K} \text { comp. }=\frac{1}{2}(\mathrm{Kc}+\mathrm{Ka})
$$

Donde:

Kc: Coeficiente de conocimiento. Se obtiene multiplicando la autovaloración del propio experto sobre sus conocimientos del tema en una escala del 0 al 10, por 0.1.

Ka: Coeficiente de argumentación. Es la suma de los valores del grado de influencia de cada una de las fuentes de argumentación (Ver Tabla 1). 
Tabla 1. Valores del grado de influencia de las fuentes de argumentación.

\begin{tabular}{|l|c|c|c|}
\hline \multicolumn{1}{|c|}{ Fuentes de Argumentación } & Alto & Medio & Bajo \\
\hline Análisis teóricos realizados por usted & 0.3 & 0.2 & 0.1 \\
\hline Experiencia obtenida & 0.5 & 0.4 & 0.2 \\
\hline Trabajos de autores nacionales que conoce & 0.05 & 0.04 & 0.03 \\
\hline Trabajos de autores extranjeros que conoce & 0.05 & 0.04 & 0.03 \\
\hline Conocimientos propios sobre el estado del tema & 0.05 & 0.04 & 0.03 \\
\hline Su intuición & 0.05 & 0.04 & 0.03 \\
\hline
\end{tabular}

Fuente: Cortés e Iglesias, 2005.

Se considera que:

- La competencia del experto es alta si $\mathrm{K}$ comp $>0.8$

- La competencia del experto es media si 0.5 $<\mathrm{K}$ comp $\leq 0.8$

- La competencia del experto es baja si $\mathrm{K}$ comp $\leq 0.5$

La Tabla 2 muestra el cálculo del coeficiente de competencia para los ocho miembros del equipo de trabajo. Se observa que todos se encuentran en el rango de clasificación entre alta y media, lo cual se considera adecuado. Este equipo participa en todas las etapas de la investigación y toma las decisiones referentes a la evaluación y mejora de la calidad percibida del servicio bancario.

Paso 3: Capacitación al equipo de trabajo

En este paso se realiza una capacitación a los miembros del equipo de trabajo con el

Tabla 2. Coeficiente de competencia de los expertos.

\begin{tabular}{|c|c|c|c|c|}
\hline Expertos & Kc & Ka & $\begin{array}{c}\text { Coeficiente de } \\
\text { competencia }\end{array}$ & $\begin{array}{c}\text { Calificación de } \\
\text { la competencia }\end{array}$ \\
\hline 1 & 0.9 & $0.3+0.5+2(0.04)+0.05+0.04=0.97$ & 0.935 & Alta \\
\hline 2 & 0.6 & $0.2+0.5+0.03+3(0.04)=0.85$ & 0.725 & Media \\
\hline 3 & 0.9 & $0.2+0.5+0.04+3(0.05)=0.89$ & 0.895 & Alta \\
\hline 4 & 0.7 & $0.3+0.5+0.03+0.04+0.05+0.04=0.96$ & 0.83 & Alta \\
\hline 5 & 0.8 & $0.3+0.5+0.03+0.04+0.05+0.04=0.96$ & 0.88 & Media \\
\hline 6 & 0.7 & $0.2+0.5+0.04+0.03+0.04+0.05=0.86$ & 0.78 & Media \\
\hline 7 & 0.8 & $0.2+0.4+4(0.04)=0.76$ & 0.78 & Media \\
\hline 8 & 0.7 & $0.2+0.5+3(0.05)+0.04=0.89$ & 0.795 & \\
\hline
\end{tabular}


objetivo de que conozcan y entiendan de manera colectiva los principales elementos relacionados con la calidad de los servicios y los modelos para su medición, la gestión por procesos, así como las herramientas y técnicas a emplear. Para ello se realizan cuatro sesiones de trabajo en forma de talleres. Ha de destacarse que la Directora de la sucursal es graduada de Ingeniería Industrial, lo cual constituye una ventaja en todo este proceso.

Paso 4: Información a todos los niveles de los objetivos del estudio

Se informa a los empleados de todos los niveles sobre la realización del estudio y los objetivos que se persiguen con el mismo. Se explica la necesidad de la participación activa de los trabajadores en la investigación buscando el compromiso y contribución de todos para un desarrollo exitoso. Se da a conocer el equipo de trabajo en reuniones con los trabajadores de la sucursal y los beneficios a obtener con la investigación que se realiza.

\subsection{Etapa II: Selección y descripción del proceso de servicio bancario a analizar}

Paso 5. Selección del proceso de servicio bancario objeto de estudio

Para la selección del proceso de servicio bancario objeto de estudio, el equipo de trabajo decide inicialmente estratificar las quejas recibidas en los últimos tres trimestres del año 2013, clasificándolas en quejas referentes a los servicios de caja y aquellas relativas a los servicios del área comercial. De este análisis se concluye que alrededor del $80 \%$ de las quejas corresponden a los servicios del área comercial.
Se realiza además un análisis de Pareto con el objetivo de identificar en cuáles servicios de esta área se identifican la mayor cantidad de quejas, y se observa que el $66.67 \%$ de las quejas están relacionadas con el servicio de solicitud, otorgamiento y entrega de créditos. Además, este servicio del área comercial es el más demandado por los clientes representando el $52.08 \%$ del total de los servicios solicitados durante el tercer trimestre del año 2013. Dichos resultados se muestran en la Figura 2. Este análisis evidencia que los esfuerzos se deben centrar en este servicio.
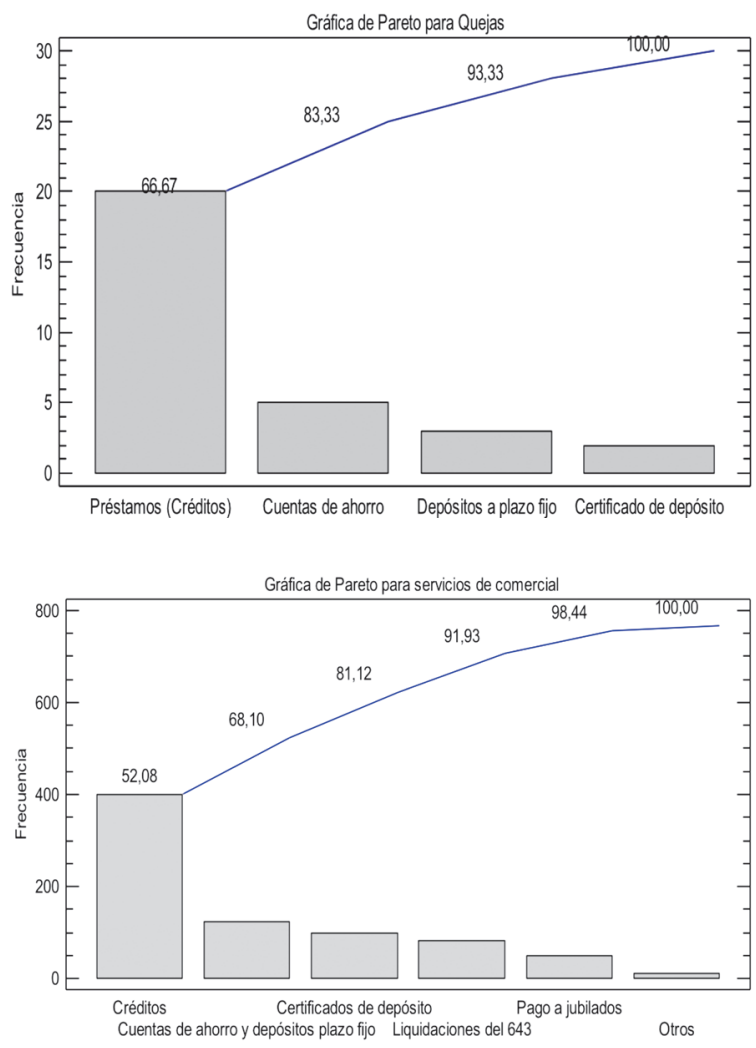

Figura 2. Diagramas de Pareto para quejas del área comercial y para servicios de esta área solicitados en el tercer trimestre del año 2013 
Paso 6. Descripción del proceso de servicio bancario

En este paso se procede a la documentación del subproceso de Solicitud, Otorgamiento y Entrega de Créditos. Como resultados de este paso se obtiene lo siguiente:

- Diagrama SIPOC (Supplier, inputs, process, outputs, customer).

- Ficha de proceso.

- Diagrama de flujo "quién-qué".

- Indicadores: Para determinar los indicadores del subproceso objeto de estudio se realiza un método Delphi por rondas en el que quedan establecidos los siguientes indicadores según orden de importancia: nivel de calidad del servicio, tiempo de ciclo y porcentaje de quejas y reclamaciones de clientes. A partir de ello se elaboran las fichas de los indicadores como una vía para formalizarlos. Los patrones de referencia se determinan por consenso y se relacionan con una escala cualitativa ordinal (Excelente, adecuado e insuficiente) asociando esta a una escala Likert de tres niveles para evaluar de manera integral el proceso. Una vez determinado el orden de importancia de los indicadores a través del método Delphi, los expertos establecen en consenso el peso para cada indicador en la evaluación del proceso. La Tabla 3 muestra un resumen de los indicadores.

\subsection{Etapa III: Medición y análisis de la calidad de servicio bancario percibida por el cliente}

Paso 7. Diseño o selección del instrumento a utilizar para medir el nivel de calidad de servicio percibida

Como se comentó anteriormente, se selecciona el cuestionario Servqual para medir el nivel de calidad de servicio percibido en las

Tabla 3. Principales características de los indicadores.

\begin{tabular}{|c|c|c|c|}
\hline Indicador & Forma de cálculo & Patrón de referencia & Frecuencia \\
\hline $\begin{array}{l}\text { Nivel de } \\
\text { calidad del } \\
\text { servicio }\end{array}$ & $\begin{array}{l}\text { PS }=P-E \\
\text { Donde: } \\
\text { PS: Puntuación de Servqual } \\
\text { P: Percepciones del servicio } \\
\text { E: Expectativas del servicio }\end{array}$ & $\begin{array}{c}\text { PS }>0 \text { Existe un excelente nivel de } \\
\text { calidad (Excelente) } \\
\text { PS }=0 \text { Existe calidad en el servicio } \\
\text { (Adecuado) } \\
\text { PS }<0 \text { Existe falta de calidad } \\
\text { (Insuficiente) }\end{array}$ & Trimestral \\
\hline $\begin{array}{l}\text { Tiempo de } \\
\text { ciclo }\end{array}$ & $\begin{array}{l}\quad \mathrm{C}_{\mathrm{ps}}=\mathrm{C}_{\mathrm{pk}}=\frac{\mathrm{ES}-\mu}{3 \sigma} \\
\text { Donde: } \\
\mathrm{C}_{\mathrm{pk}} \text { : Índice de capacidad real del proceso } \\
\text { ES: Especificación superior } \\
\mu \text { : Media del proceso } \\
\sigma \text { : Desviación estándar del proceso } \\
\text { Nota: } \\
\checkmark \text { El proceso debe ser estable. } \\
\checkmark \text { La característica de calidad debe } \\
\text { distribuirse normalmente. } \\
\checkmark \text { La desviación estándar del proceso se } \\
\text { debe conocer. }\end{array}$ & $\begin{array}{c}\mathrm{C}_{\mathrm{pk}}>1.25 \text { Excelente } \\
\mathrm{C}_{\mathrm{pk}}=1.25 \text { Adecuado } \\
\mathrm{C}_{\mathrm{pk}}<1.25 \text { Insuficiente }\end{array}$ & Trimestral \\
\hline $\begin{array}{l}\text { Por ciento de } \\
\text { quejas y } \\
\text { reclamaciones } \\
\text { de clientes }\end{array}$ & $\frac{\text { Total de quejas }}{\text { Total de clientes atendidos }} \times 100$ & $\begin{array}{c}<1 \% \text { Excelente } \\
\leq 1 \% \text { y }<2 \% \text { Adecuado } \\
\geq 2 \% \text { Insuficiente }\end{array}$ & Trimestral \\
\hline
\end{tabular}


instituciones bancarias. Se tomará como caso de estudio específicamente las percepciones de los clientes del Subproceso de Solicitud, Otorgamiento y Entrega de Créditos.

Paso 8. Aplicación del instrumento de medición de calidad de servicio percibida

Para el estudio que se realiza se define como población (N) la cantidad de clientes que visitaron la sucursal para recibir el servicio de Solicitud, Otorgamiento y Entrega de Créditos en el segundo semestre del año 2013, y que asciende a 630. Por tanto, el modo de cálculo a utilizar para determinar el tamaño de muestra necesario es para población finita $(<100000)$ y varianza desconocida. Los parámetros tenidos en cuenta se muestran en la Tabla 4. El total de clientes a encuestar es de 84. Como tipo de muestreo a utilizar se selecciona el muestreo aleatorio simple, se encuestan aleatoriamente clientes que reciben este servicio en la sucursal durante el mes de enero de 2014.

Paso 9. Procesamiento de la información y análisis estadístico de los resultados de la medición de calidad de servicio percibida por el cliente

El procesamiento de los cuestionarios se realiza con la ayuda del Software Estadístico SPSS Versión 19.0. Para probar la fiabilidad del instrumento se emplea el coeficiente Alpha de Cronbach. La Tabla 5 muestra los valores del coeficiente Alpha de Cronbach para cada una de las dimensiones del cuestionario y para la propia escala en su conjunto.

La fiabilidad del instrumento se considera adecuada, puesto que todos los valores del Alpha de Cronbach se encuentran por encima de 0.7 .

Análisis de las percepciones de los clientes

Para este análisis se toman en cuenta únicamente las percepciones de los clientes y se considera en desacuerdo a los encuestados que marcaron del 1 al 3 en la escala Likert de 7 niveles, indiferente ( $\mathrm{ni}$ de acuerdo ni en desacuerdo) a los que marcaron 4 y de acuerdo a los que marcaron del 5 al 7. Las

Tabla 4. Datos para la determinación del tamaño de muestra.

\begin{tabular}{|c|c|c|}
\hline Forma de cálculo & \multicolumn{2}{|r|}{ Datos } \\
\hline \multirow{6}{*}{$\mathbf{n}=\frac{\mathbf{N P q}}{\frac{(\mathbf{N}-1) \mathbf{B}^{2}}{\mathbf{z}^{2}}+\mathbf{P q}}$} & Nivel de confianza (NC) & Para un NC de $95 \%$ el valor de z es 1.96 \\
\hline & Población $(\mathrm{N})$ & 105 \\
\hline & $\begin{array}{c}\text { Proporción muestral o su } \\
\text { estimado }(P)\end{array}$ & 0.5 \\
\hline & Error permisible (B) & 0.10 \\
\hline & q & $1-p$ \\
\hline & Tamaño de la muestra & 84 \\
\hline
\end{tabular}

Tabla 5. Alfa de Cronbach para el cuestionario Servqual en su conjunto y por dimensiones.

\begin{tabular}{|c|c|c|c|c|c|c|} 
Cuestionario & $\begin{array}{c}\text { Elementos } \\
\text { tangibles }\end{array}$ & Fiabilidad & $\begin{array}{c}\text { Capacidad } \\
\text { de } \\
\text { respuesta }\end{array}$ & Seguridad & Empatía & $\begin{array}{c}\text { Escala en } \\
\text { su } \\
\text { conjunto }\end{array}$ \\
\hline Servqual & 0.801 & 0.865 & 0.824 & 0.813 & 0.875 & $\mathbf{0 . 8 3 0}$ \\
\hline
\end{tabular}


mayores insatisfacciones de los clientes están relacionadas con el tiempo de servicio, donde más del $80 \%$ de los encuestados manifestó estar en desacuerdo con la rapidez del servicio que se presta en la sucursal. Lo anterior corrobora lo obtenido en el diagrama de Pareto en el paso 1 del procedimiento donde la principal queja de los clientes se relaciona con la demora del servicio. En orden le siguen las insatisfacciones relativas al planteamiento «la sucursal bancaria realiza bien el servicio la primera vez», donde el $44.9 \%$ de los encuestados manifestó estar en desacuerdo.

\section{Análisis de expectativas y percepciones}

Para verificar si existen diferencias estadísticamente significativas entre las expectativas de los clientes y sus percepciones en relación al servicio objeto de estudio se utiliza la Prueba W de Mann-Whitney (Wilcoxon) para comparar medianas. La Tabla 6 muestra de manera general las pruebas de hipótesis planteadas. Esto se realiza para cada una de las 22 declaraciones con ayuda del Software Estadístico Statgraphics Centurion.

Los resultados del análisis estadístico se muestran en la Tabla 7 donde se concluye que existen diferencias estadísticamente significativas entre las expectativas de los clientes y sus percepciones por declaraciones y se concluye, además, que para todos los casos las expectativas superan las percepciones dado que el valor-P es menor que el nivel de significación $\alpha=0.05$. Este hecho evidencia que existe un insuficiente nivel de calidad del servicio.

Para calcular la puntuación Servqual como se muestra en (1) y determinar las brechas existentes entre expectativas y percepciones se utilizó el promedio de los valores obtenidos para cada uno de los 22 atributos. Las principales brechas de insatisfacción identificadas en la calidad del servicio para cada una de las cinco dimensiones del cuestionario Servqual se muestran en la Tabla 8.

Tabla 6. Prueba W de Mann-Whitney para verificar diferencias estadísticamente significativas entre expectativas y percepciones.

\begin{tabular}{|c|c|c|c|}
\hline \multicolumn{2}{|c|}{$\begin{array}{c}\text { Comparación de medianas para verificar si existen } \\
\text { diferencias significativas entre expectativas y } \\
\text { percepciones }\end{array}$} & \multicolumn{2}{|c|}{$\begin{array}{l}\text { Comparación de medianas para verificar si las } \\
\text { expectativas superan las percepciones }\end{array}$} \\
\hline \multicolumn{2}{|c|}{ Prueba de hipótesis $1(\mathrm{PH}-1)$} & \multicolumn{2}{|c|}{ Prueba de hipótesis 1 (PH-2) } \\
\hline $\begin{array}{c}\mathrm{H}_{0}: \text { mediana } 1 \\
(\text { Expectativas })= \\
\text { mediana } 2 \\
\text { (Percepciones) }\end{array}$ & $\begin{array}{c}\text { No existen } \\
\text { diferencias } \\
\text { significativas entre las } \\
\text { medianas } \\
\end{array}$ & $\begin{array}{c}\mathrm{H}_{\mathrm{o}}: \text { mediana1 } \\
(\text { Expectativas })= \\
\text { mediana } 2 \\
\text { (Percepciones) }\end{array}$ & $\begin{array}{c}\text { No existen } \\
\text { diferencias } \\
\text { significativas entre } \\
\text { las medianas } \\
\end{array}$ \\
\hline $\begin{array}{l}\mathrm{H}_{1}: \text { mediana1 } \\
(\text { Expectativas } \neq \\
\text { mediana } 2 \\
\text { (Percepciones) }\end{array}$ & $\begin{array}{c}\text { Existen diferencias } \\
\text { significativas entre las } \\
\text { medianas }\end{array}$ & $\begin{array}{c}\mathrm{H}_{1} \text { : mediana1 } \\
\text { (Expectativas }>\text { mediana } \\
2 \text { (Percepciones) }\end{array}$ & $\begin{array}{c}\text { La mediana de la } \\
\text { primera muestra es } \\
\text { significativamente } \\
\text { mayor que la } \\
\text { mediana de la } \\
\text { segunda }\end{array}$ \\
\hline & tica: valor- $\mathrm{P}<\alpha$ & el nivel de signific & sión \\
\hline
\end{tabular}


Tabla 7. Resumen estadístico de la aplicación del cuestionario Servqual.

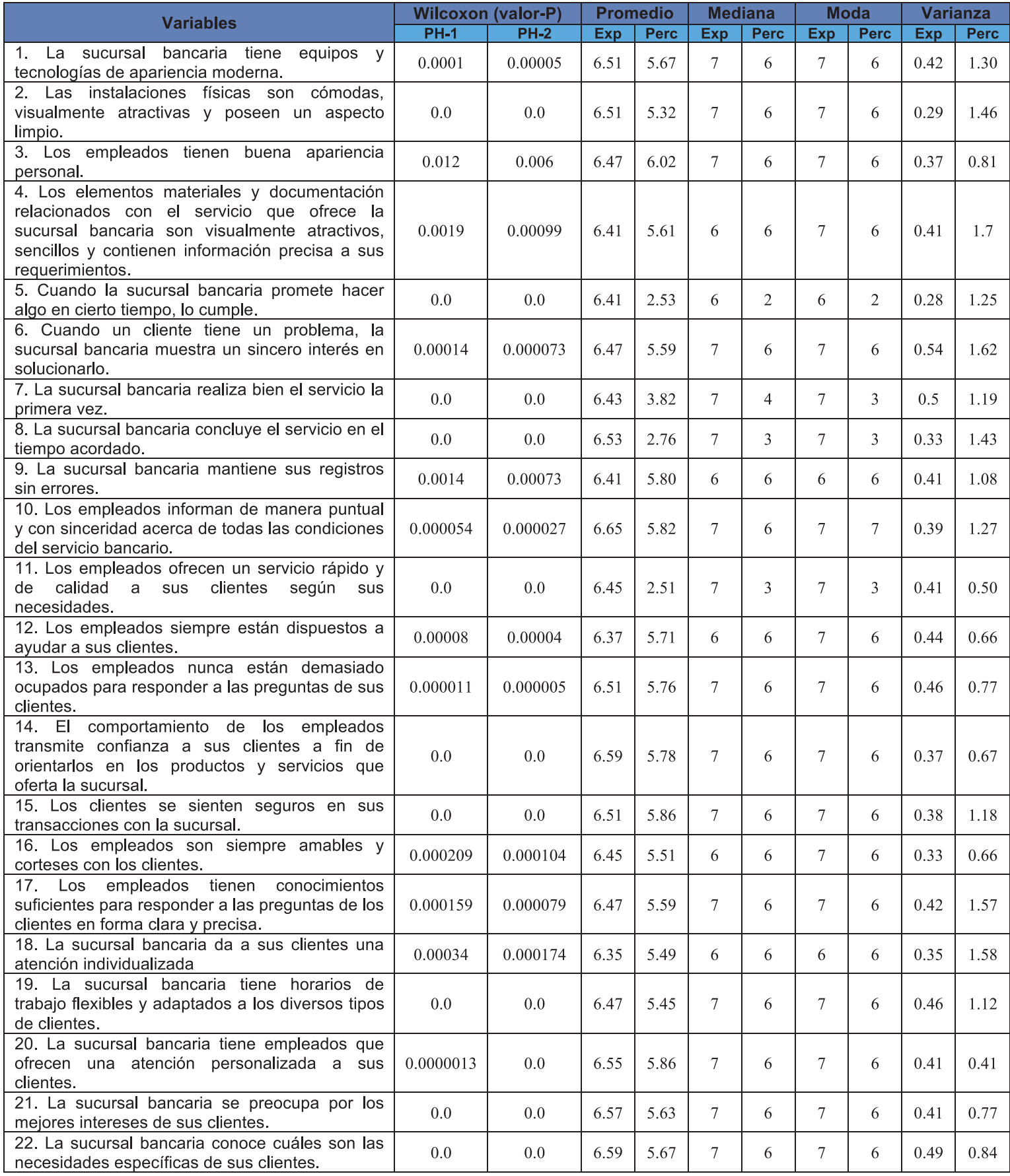


Tabla 8. Principales brechas de insatisfacción en la calidad del servicio por dimensión.

\begin{tabular}{|c|c|}
\hline $\begin{array}{l}\text { Dimensiones del } \\
\text { Cuestionario } \\
\text { Servqual }\end{array}$ & Principales brechas de insatisfacción en la calidad del servicio percibida \\
\hline $\begin{array}{c}\text { Elementos } \\
\text { tangibles }\end{array}$ & $\begin{array}{l}\checkmark \quad \text { Las instalaciones físicas de la sucursal bancaria son cómodas, visualmente atractivas y } \\
\text { poseen un aspecto limpio ( }-1.19) \text {. } \\
\checkmark \quad \text { La sucursal bancaria tiene equipos y tecnologías de apariencia moderna }(-0.84) \text {. }\end{array}$ \\
\hline Fiabilidad & $\begin{array}{ll}\checkmark & \text { Cuando la sucursal bancaria promete hacer algo en cierto tiempo, lo cumple (-3.88). } \\
\checkmark & \text { La sucursal bancaria concluye el servicio en el tiempo acordado (-3.77) } \\
\checkmark & \text { La sucursal bancaria realiza bien el servicio la primera vez (-2.61) }\end{array}$ \\
\hline $\begin{array}{l}\text { Capacidad de } \\
\text { respuesta }\end{array}$ & $\begin{array}{l}\checkmark \text { Los empleados de la sucursal bancaria ofrecen un servicio rápido y de calidad a sus } \\
\text { clientes según sus necesidades }(-3.94) \\
\checkmark \quad \text { Los empleados de la sucursal bancaria informan de manera puntual y con sinceridad } \\
\text { acerca de todas las condiciones del servicio bancario }(-0.83) \text {. }\end{array}$ \\
\hline Seguridad & $\begin{array}{l}\checkmark \text { Los empleados de la sucursal bancaria son siempre amables y corteses con los } \\
\text { clientes }(-0.94) \\
\checkmark \text { Los empleados de la sucursal bancaria tienen conocimientos suficientes para } \\
\text { responder a las preguntas de los clientes en forma clara y precisa }(-0.88)\end{array}$ \\
\hline & $\begin{array}{l}\checkmark \quad \text { La sucursal bancaria tiene horarios de trabajo flexibles y adaptados a los diversos tipos } \\
\text { de clientes }(-1.02) \\
\checkmark \quad \text { La sucursal bancaria se preocupa por los mejores intereses de sus clientes }(-0.94)\end{array}$ \\
\hline
\end{tabular}

De manera general puede afirmarse que en ninguno de los casos las percepciones sobrepasan las expectativas. Las principales brechas se encuentran en los atributos relacionados con la duración del tiempo de servicio y cuyas declaraciones se muestran a continuación:

- Los empleados de la sucursal bancaria ofrecen un servicio rápido y de calidad a sus clientes según sus necesidades (-3.94).

- Cuando la sucursal bancaria promete hacer algo en cierto tiempo, lo cumple (-3.88).

- La sucursal bancaria concluye el servicio en el tiempo acordado (-3.77).

- La sucursal bancaria realiza bien el servicio la primera vez (-2.61).

Análisis por dimensión y nivel global de calidad del servicio

Para determinar la importancia que tienen cada una de las dimensiones del cuestionario Servqual en la evaluación global de la calidad del servicio se le solicita al cliente encuestado que ordene las cinco dimensiones utilizando una escala del 1 al 5 de acuerdo con la importancia que tenga para éste, donde 5 se considera como más importante y 1 menos importante. Para este análisis se utiliza la moda como parámetro estadístico, lo cual se muestra a continuación:

- Elementos tangibles (Moda: 1).

- Fiabilidad (Moda: 5).

- Capacidad de respuesta (Moda: 4).

- Seguridad (Moda: 3).

- Empatía (Moda: 2).

Una vez determinado el orden de importancia, el equipo de trabajo establece en consenso el peso para cada dimensión. Esta ponderación se realiza distribuyendo 100 puntos entre las cinco dimensiones, para luego recalcular los valores de expectativas y percepciones para cada dimensión y, una vez realizado esto, volver a calcular la brecha utilizando los puntajes ponderados. En la Tabla 9 se muestran las brechas existentes entre las expectativas y las percepciones para cada una de las dimensiones 
que presenta el cuestionario Servqual en su versión original y ponderada.

Se observa en ambos casos que las percepciones no superan las expectativas, es decir, las expectativas no fueron alcanzadas. En la versión original se identifica como dimensión más crítica la Fiabilidad con una brecha de -2.35, seguida por la Capacidad de Respuesta (-1.51). Las dimensiones menos críticas son Elementos Tangibles y Seguridad, ambas con una brecha de -0.82. No obstante, las percepciones en ambas dimensiones están todavía por debajo de las expectativas. En el caso de la versión ponderada, igualmente se obtienen como dimensiones más críticas la Fiabilidad y la Capacidad de Respuesta. Se identifica en este caso como la dimensión menos crítica los Elementos Tangibles, seguida de la Empatía.

Por último se calcula un índice global de calidad de servicio a partir de la realización de una media de los valores medios, tanto de las expectativas como de las percepciones de cada una de las dimensiones, cuyos resultados se muestran en la Tabla 7 antes mencionada, donde se aprecia que es de - 1.27 para la versión original y en el caso de la versión ponderada de -33.1. Este indicador, al ser negativo, evidencia que las expectativas del cliente no están siendo cubiertas por la percepción que este tiene del servicio que se le está brindando. A medida que este indicador se acerque a cero (al venir de un valor negativo), puede entenderse que la percepción del servicio se va acercando a las expectativas que el cliente tiene.

\subsection{Etapa IV: Mejoramiento continuo del proceso de servicio bancario objeto de estudio}

\section{Paso 10. Planificar}

Identificación y análisis de la situación

Las acciones de mejora se enfocan hacia las principales brechas de insatisfacción identificadas en la calidad del servicio percibido por el cliente, las cuales se relacionan, como se comentó anteriormente, con la duración del tiempo de servicio. Para investigar las causas por las cuales no se ofrece un servicio rápido al cliente se utiliza el diagrama causa-efecto, específicamente el de estratificación de causas puesto que es un método gráfico que permite relacionar el problema con todas las causas que posiblemente lo generan. Para ello se acude al equipo de trabajo y se realizan además

Tabla 9. Brechas según dimensiones del cuestionario Servqual.

\begin{tabular}{|c|c|c|c|c|c|c|c|}
\hline \multirow{2}{*}{ Dimensiones } & \multirow{2}{*}{ Ponderación } & \multicolumn{3}{|c|}{ Puntaje obtenidos } & \multicolumn{3}{|c|}{ Puntajes ponderados } \\
\hline & & Expectativas & Percepciones & Brechas & Expectativas & Percepciones & Brechas \\
\hline $\begin{array}{c}\text { Elementos } \\
\text { tangibles }\end{array}$ & 5 & 6.47 & 5.65 & -0.82 & 32.35 & 28.25 & -4.1 \\
\hline Fiabilidad & 40 & 6.45 & 4.1 & -2.35 & 258 & 164 & -94 \\
\hline $\begin{array}{c}\text { Capacidad } \\
\text { de respuesta }\end{array}$ & 30 & 6.49 & 4.95 & -1.51 & 194.7 & 148.5 & -46.2 \\
\hline Seguridad & 15 & 6.50 & 5.68 & -0.82 & 97.5 & 85.2 & -12.3 \\
\hline Empatía & 10 & 6.50 & 5.62 & -0.88 & 65 & 56.2 & -8.8 \\
\hline Total & 100 & $\begin{array}{r}\text { Índice Glob } \\
\text { del S }\end{array}$ & $\begin{array}{l}\text { de Calidad } \\
\text { vicio }\end{array}$ & -1.27 & $\begin{array}{l}\text { Índice Glok } \\
\text { del Servicic }\end{array}$ & $\begin{array}{l}\text { I de Calidad } \\
\text { (Ponderado) }\end{array}$ & -33.1 \\
\hline
\end{tabular}


entrevistas no estructuradas a trabajadores, directivos y algunos clientes de la sucursal.

Para obtener un orden de prioridad entre las causas se utiliza el método Delphi, donde se determina que existe comunidad de preferencia entre los expertos con un coeficiente $W$ de Kendall de 0.789, lo cual se considera adecuado. Atendiendo a este resultado se identifican como principales oportunidades de mejora las siguientes:

- No se realizan estudios de identificación de flujo de valor.

- No se selecciona, evalúa y capacita por competencias.

- Excesiva carga mental.

Establecimiento de las mejoras a alcanzar

A partir de la identificación de las principales oportunidades de mejora se procede al diseño de un plan mediante el uso de la técnica $5 \mathrm{~W}$ y $1 \mathrm{H}$. En este plan de mejora queda precisada la meta que se espera alcanzar con dicho plan y el responsable general. Además se establecen qué acciones se llevarán a cabo, quiénes las implantarán, qué métodos o técnicas se utilizarán para ello, por qué es necesario realizar esas acciones de mejora, dónde y cuándo se realizarán.

Para este caso en particular se define como meta: Disminuir el tiempo desde que se realiza la solicitud del crédito hasta su otorgamiento y entrega, y se define como responsable general a la directora de la sucursal. Las principales acciones de mejora que se proponen son:

1. Realizar un estudio para identificar actividades del proceso que no son necesarias.
2. Realizar un estudio de carga mental.

3. Realizar un estudio para la identificación, reglamentación, evaluación y certificación de las competencias laborales de trabajadores involucrados en el proceso.

Realizar un estudio para identificar las actividades del proceso que no son necesarias

Con el objetivo de identificar las actividades dentro del proceso que no agregan valor y que no son necesarias, se aplica la metodología de Proceso Esbelto descrita por Gutiérrez y De la Vara [24]. La Tabla 10 muestra las actividades que se dan a lo largo del Subproceso de Solicitud, Otorgamiento y Entrega de Créditos, clasificadas en: agrega valor de acuerdo a la percepción del cliente, muda 1 o muda 2 y estimación del tiempo que consume cada actividad. Las mudas implican eliminar 0 perder tiempos, movimientos y errores en un proceso. Las mudas tipo 1 son las que no crean valor pero que actualmente son necesarias para el desarrollo del servicio y las mudas tipo 2 son aquellas que no crean valor de acuerdo al cliente y que pueden y deben ser eliminadas.

Para la estimación del tiempo de cada actividad se realiza un cronometraje. Como muestra inicial se toman treinta observaciones en cada actividad y luego se realiza el cálculo del número total de observaciones a realizar a partir de las treinta primeras según el criterio de Marsán [25]. Para la verificación del supuesto de normalidad de los datos para cada una de las actividades del proceso se hace uso de la prueba Kolmogorov-Smirnov. Para todos los casos se cumple que el valor-P es mayor que $\alpha=0.05$, hecho que evidencia que los datos provienen de una distribución normal con 95\% de confianza.

Para verificar la estabilidad de los datos se utilizan gráficos de control de individuales, 
Tabla 10. Clasificación y tiempo de duración de las actividades.

\begin{tabular}{|c|l|c|c|}
\hline No. & \multicolumn{1}{|c|}{ Actividad } & Tiempo (minutos) & Muda \\
\hline 1 & Solicitud de la planilla y orientación al cliente & 19.95 & Agrega valor \\
\hline 2 & Entrega de planillas al cliente & 10.65 & Agrega valor \\
\hline 3 & Llenado de planilla por parte del cliente & $\begin{array}{c}\text { Actividad que depende de la rapidez con } \\
\text { que el cliente realice esta gestión }\end{array}$ \\
\hline 4 & $\begin{array}{l}\text { Entrega de la planilla por parte del cliente al } \\
\text { banco }\end{array}$ & 6.23 & Agrega valor \\
\hline 5 & Revisión de la planilla & 40.66 & 1 \\
\hline 6 & Solicitud de referencia & 39.70 & 2 \\
\hline 7 & Análisis de riesgo & 120.19 & 1 \\
\hline 8 & Aprobación o denegación del crédito & 26.18 & Agrega valor \\
\hline 9 & Formulación del crédito & 19.32 & 1 \\
\hline 10 & Entrega del crédito & 15.93 & Agrega valor \\
\hline
\end{tabular}

los cuales son útiles para detectar cambios tanto en la media como en la dispersión del proceso. Del análisis de las cartas de control de individuales para cada una de las actividades del proceso se puede concluir que existe regularidad estadística, así como baja dispersión de los datos dado que todos los puntos se encuentran dentro de los límites de control y no se observan patrones especiales. El procesamiento de los datos se realiza con el paquete de programa Statgraphics Centurion para todos los casos.

A modo de ejemplo se muestra en la Figura 3 la prueba Kolmogorov-Smirnov y el gráfico de control correspondiente a la actividad 6 "Solicitud de referencia".

Con la ayuda del equipo de trabajo y la realización de un profundo análisis de cada una de las actividades del proceso, se toma la decisión de eliminar la actividad 6 clasificada como Muda 2, la cual no crea valor para el cliente y no es necesaria. La solicitud de referencia se considera Muda 2 dado que esta actividad se realiza para corroborar la veracidad de lo establecido en la planilla entregada por el cliente, en la cual en uno de sus apartados la entidad empleadora debe informar al banco todos los descuentos que tiene el trabajador. $\mathrm{Ha}$

\begin{tabular}{|c|c|}
\hline \multicolumn{2}{|c|}{$\begin{array}{c}\text { Pruebas Bondad-de-Ajuste para la } \\
\text { Actividad 6 } \\
\text { Prueba de Kolmogorov-Smirnov }\end{array}$} \\
\hline & Distribución Normal \\
\hline DPLUS & 0.0969279 \\
\hline DMINUS & 0.0965993 \\
\hline DN & 0.0969279 \\
\hline Valor-P & 0.940693 \\
\hline \multicolumn{2}{|c|}{} \\
\hline
\end{tabular}
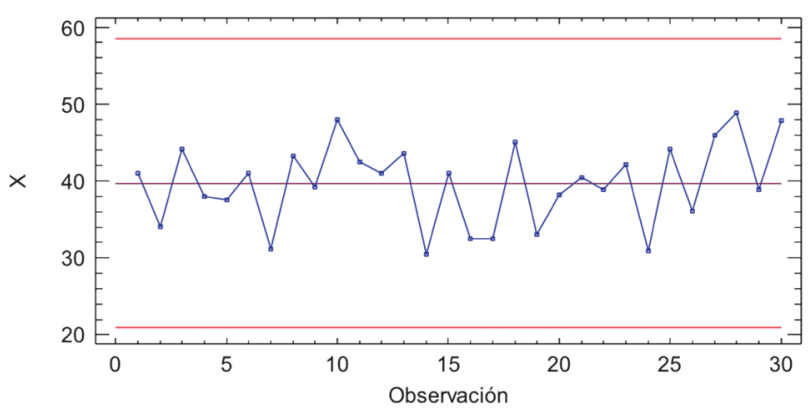

Figura 3. Prueba de bondad de ajuste y gráfico de control de individuales para la actividad 6.
$\mathrm{LSC}=58,49$ CTR $=39,70$ $\mathrm{LIC}=20,92$ 
de destacarse que cuando se realiza el análisis del crédito o de riesgo por parte de los gestores de negocio, estos están obligados a verificar la información contenida en la planilla en lo que se refiere a la capacidad de pago del cliente y de los fiadores, de manera que se realiza la misma verificación dos veces. Otro aspecto importante a destacar es que la actividad de solicitud de referencia se realiza en presencia del cliente y de los fiadores, lo que genera demoras en el servicio. Todo lo anterior evidencia la necesidad de eliminar dicha actividad, cuya duración es como promedio de $40 \mathrm{~min}$.

Una vez propuestas las acciones de mejora se procede a su implantación (Paso 11) para luego verificar la efectividad de las mismas (Paso 12). Para ello se recomienda evaluar el proceso a través de los indicadores propuestos. En función de los resultados de la comprobación anterior se realizan las correcciones necesarias (ajuste) o se convierten las mejoras alcanzadas en una forma estabilizada de ejecutar el proceso (actualización) (Paso 13).

\section{CONCLUSIONES}

El procedimiento diseñado para la evaluación de la calidad percibida de los servicios bancarios compuesto de cuatro etapas y trece pasos garantiza la evaluación y mejora de la calidad percibida mediante la escala Servqual, con un enfoque de gestión por procesos y mejora continua, haciendo uso de diversas herramientas.

La implementación del procedimiento en el Servicio de Solicitud, Otorgamiento y Entrega de Créditos permitió identificar las principales brechas de insatisfacción en la calidad del servicio percibido por los clientes, resultados que facilitaron la toma de decisiones para proyectar acciones de mejora orientadas a elevar la calidad del mismo.

La evaluación de la calidad percibida evidenció que para las 22 declaraciones del cuestionario Servqual, las percepciones de los clientes no superaban las expectativas y las brechas con menor índice de calidad se relacionaban con el tiempo de servicio. En relación con esto, el índice global de calidad de servicio mostró un deficiente nivel de calidad en el servicio al tomar valores negativos tanto para la versión original (-1.27) como para la ponderada (-33.1), donde las dimensiones más críticas son la Fiabilidad y la Capacidad de Respuesta.

Se propuso un conjunto de acciones encaminadas a la mejora en función de las principales brechas de insatisfacción identificadas en la calidad del servicio. Se destaca la realización de un estudio para identificar actividades que no agregan valor y que no son necesarias mediante la metodología de proceso esbelto, lo que permitió la disminución del tiempo de servicio en 40 minutos como promedio.

La gestión de la calidad de los servicios bancarios desde la percepción de los clientes constituye una manera de conseguir la satisfacción de estos, objetivo permanente en organizaciones de este tipo, al considerarse la calidad del servicio como antecedente de la satisfacción.

\section{REFERENCIAS BIBLIOGRÁFICAS}

[1] González, Z. (2001). El reto de la calidad del servicio financiero en la Comunidad Autónoma de Canarias. Santa Cruz de Tenerife, Canarias: Ed. Fundación FYDECaja Canarias. 
[2] Cobra, M. (2002). Marketing de servicios: Estrategias para el turismo, finanzas, salud y comunicación (2 Ed.). P. 255. Recuperado de http://umecit. metabiblioteca.org/cgi-bin/koha/opacdetail.pl?biblionumber=40.

[3] Dávila, J.A. y Flórez, M. (2008). Calidad del servicio percibida por clientes de entidades bancarias de Castilla y León y su repercusión en la satisfacción y lealtad de la misma. PECVNIA. Revista de la Facultad de Ciencias Económicas y Empresariales de la Universidad de León (7), 105-128.

[4] Shih-Y. y Fang-K. (2006). Effects of Network Quality Attributes on Customer Adoption Intentions of Internet Banking. Total Quality Management Journal, 17 (1), 61-77.

[5] Skowron, L. y Kristensen, K. (2012). The impact of the recent banking crisis on customer loyalty in the banking sector: Developing versus developed countries. Total Quality Management Journal, 24(6), $480-497$.

[6] Morillo, M. (2009). La calidad en el servicio y la satisfacción del usuario en instituciones financieras del municipio Libertador del estado Mérida. Revista Economía, XXXIV(27), 199-223.

[7] Gray, J. y Harvey, T. (2009). El valor de la calidad en los servicios bancarios. México: Ed. LIMUSA, p. 239.

[8] Cronin, J. y Taylor, S. (1992). Measuring Service Quality: A Reexamination and Extension. Journal of Marketing, 56(3), 55-68.
[9] Grönroos, C. (1984). A Service Quality Model and Its Marketing Implications. European Journal of Marketing, 18(4), 36-44.

[10] Karatepe, O.M., Yavas, U. y Babakus, E. (2005). Measuring Service Quality of Banks: Scale Development and Validation. Journal of Retailing and Consumer Services, 12(5), 373-383.

[11] Morillo, M. y Rivas, D. (2011). Medición de la calidad del servicio en las instituciones financieras a través de la escala de Servqual. Revista Contaduría y Administración, (234), 101-130.

[12] Chi Cui, C., Lewis, B.R. y Park, W. (2003). Service Quality Measurement in the Banking Sector in South Korea. International Journal of Bank Marketing, 21(4), 191-201.

[13] Sharma, A. y Mehta, V. (2004). Service Quality Perceptions in Financial Services A Case Study of Banking Services. Journal of Services Research, 4(2), 205-223.

[14] Colmenares, L.C. (2008). Evaluación de la calidad del servicio de atención al cliente prestado por el BBVA Banco Provincial, Oficina Rómulo Gallegos de Barquisimeto Estado Lara (Tesis de especialidad inédita). Universidad Centroccidental Lisandro Alvarado, Estado Lara, Venezuela.

[15] Mejías, A. y Manrique, S. (2011). Dimensiones de la satisfacción de clientes bancarios universitarios: Una aproximación mediante el análisis de factores. Revista Ingeniería Industrial, 32(1), 43-47. 
[16] Aldlaigan, A. y Buttle, F. (2002). SYSTRASQ: A New Measure of Bank Service Quality. International. Journal of Service Industry Management, 13(4), 362 - 381.

[17] Martínez, M.D.J. (2006). La Calidad del Servicio Percibida en Entornos Virtuales de Formación Superior (Tesis de doctorado inédita). Universidad de Barcelona, Barcelona, España.

[18] Parasuraman, A., Berry, L. y Zeithaml, V. (1988). SERVQUAL: A multiple-Item Scale for Measuring Consumer Perceptions of Service Quality. Journal of Retailing, 64(1), 12-37.

[19] Díaz, Y. y Pons, R. (2009). Modelización y procedimiento de evaluación de la calidad de servicios percibida en empresas cubanas de servicios. Revista Observatorio de la Economía Latinoamericana, (122).

[20] Moreno, M. (2010). Procedimiento para la medición de la calidad percibida, mejoramiento y control de los servicios de Desoft S.A. Aplicación en la División Desoft Villa Clara. En V Taller de Calidad en las Tecnologías de la Información y las Comunicaciones. La Habana, Cuba.
[21] Curbelo, D. (2013). Procedimiento para la evaluación de la calidad percibida de servicios de asistencia de salud. Caso de estudio: Hospital Provincial de Cienfuegos (Tesis de maestría inédita). Universidad de Cienfuegos, Cienfuegos, Cuba.

[22] Beltrán, J., Carmona, M., Carrasco, R., Rivas, M. y Tejedor, F. (2002). Guía para una gestión basada en procesos. España: Instituto Andaluz de Tecnología, Ed. Berekintza, p.141.

[23] Cortés, M. e Iglesias, M. (2005). Generalidades sobre la Metodología de la Investigación. 1ra. ed. México: Ed. UNACAR, p.105.

[24] Gutiérrez, P. y De la Vara, R. (2007). Control Estadístico de la Calidad y Seis Sigma. México, D.F.: Ed. McGraw-Hill, p. 636.

[25] Marsán, J., Cuesta, A., García, C. y Padilla, C. (2011). Organización del trabajo. Estudios de tiempos. La Habana, Cuba: Ed. Félix Varela. 